\title{
Estabilidad política, terrorismo y medios de comunicación. Un análisis de la incidencia de percepciones mediáticas en el turismo receptivo
}

\section{Political stability, terrorism and media. An analysis of the effect of media perceptions in receptive tourism}

Angel Torres-Toukoumidis*, Luis Romero-Rodríguez**, Amor Pérez-Rodríguez***, Ignacio Aguaded****

\author{
* Universidad Politécnica Salesiana, Ecuador \\ ** ESAI Business School, Universidad Espíritu Santo, Ecuador \\ $* * *$ Universidad de Huelva, España \\ $* * * *$ Universidad de Huelva, España
}

Resumen

\begin{abstract}
La presente investigación analiza la relación entre atentados terroristas, inestabilidad política y el discurso de los medios de comunicación y de agencias internacionales de noticias sobre el turismo receptivo, tomando como referencia países que presentaron diversas situaciones de conmoción interna entre 2012 y 2015: Egipto, Túnez, Venezuela y España. Para ello, se combinaron metodologías cuantitativas a partir de datos oficiales de organismos internacionales con el análisis del discurso periodístico de 4 agencias de noticias y 4 medios internacionales a través del uso de la herramienta informática MAXQDA (v. 11.0.11). Los resultados demuestran que la información transmitida por los medios y agencias internacionales sobre los atentados terroristas tienen mayor efecto sobre el turismo que aquellas referidas a la inestabilidad política.
\end{abstract}

Palabras clave: Estudio comparativo, terrorismo, análisis del discurso, percepción, turismo, conflictos políticos.

Abstract

The present research seeks to analyze the existing relationship between terrorist attacks, political instability, discourse from international media outlets and news agencies in receptive tourism. To accomplish this, four countries that have different internal situations will be studied in the period 20122015: Egypt, Tunisia, Venezuela and Spain combining quantitative methodologies using official data from international and national organisms, with journalistic discourse analysis from 4 international news agencies and 4 international media outlets through the use of the MAXQDA (v. 11.0.11) software tool. The results have shown that the information spread by media about terrorist attacks have more effects on tourism than those about political and economic instability.

Keywords: Comparative study, terrorism, discourse analysis, perception, tourism, political conflicts.

\section{Introducción}

Mohamed Bouazizi fue la persona que, sin saberlo, se convertiría en la chispa que iniciaría un cambio de varios regímenes autoritarios del Magreb y Medio Oriente el 17 de diciembre de 2010. Con un acto de autoinmolación en la plaza principal de Sidi Bouzid (Túnez), este vendedor ambulante decidió inconscientemente mostrarle al mundo el nivel de indignación existente en gran parte del pueblo tunecino contra la dictadura de Ben Ali. Como ya es usual por el alcance transfronterizo e inmediato de las redes sociales, la noticia le había dado la vuelta al mundo en tan solo unos segundos, haciendo imposible invisibilizar el hecho y desmovilizar las protestas contra el régimen de ese país. Las consecuencias eran 
irreversibles, Túnez dejaba de recibir $69,32 \%$ de turismo extranjero con respecto al año anterior (IEMED 2011).

La atención mundial se fijaba en las informaciones provenientes desde Túnez, pero también de las protestas colaterales en Egipto, Siria, Libia, Sahara Occidental, Líbano, Jordania, Mauritania, Sudán, Omán, Arabia Saudita, Yemen y Marruecos, países en los cuales hace apenas pocos años era impensable, o al menos intangible para el mundo occidental, la amplia penetración de Internet y mucho menos movimientos ciudadanos contrarios a sus regímenes (Soengas 2013). Los medios de comunicación adjetivaron este conjunto de acontecimientos como "Primavera Árabe" evocando a la Primavera de Praga de 1968.

Aunado a la propia inestabilidad política y bajos niveles de desarrollo socio-económico de estos países, nueve de cada 10 atentados en el mundo se producen actualmente en Asia meridional, oriente medio y la mitad septentrional de África, pero tres cuartas partes de estos ocurren en países y territorios con poblaciones mayoritariamente musulmanas (Reinares 2012).

A partir de 2012, coincidiendo con el período de mayor inestabilidad política generado por las revueltas mencionadas, España alcanzaba cifras récords en ingreso de turistas internacionales y en especial europeos, número que ha mantenido una tendencia al alza desde esas fechas según datos cruzados entre el World Turism Organization (UNWTO) y el European Institute of Mediterranean (IEMED). Mientras en 2011 el país ibérico recibía 52.676.972 visitantes internacionales, en el marco de la primavera árabe en 2012 se totalizaban 56.176 .886 , un incremento diferencial significativo de 6,64\%, que podría suponer un juego de suma cero en el turismo receptivo del mediterráneo.

\section{Seguridad e Inestabilidad política}

Sönmez (1998) describe que las organizaciones terroristas buscan realizar atentados en lugares turísticos como una herramienta para crear inestabilidad en la economía, dado que la violencia política, en cualquiera de sus formas, afecta directamente las industrias culturales, incluyendo al turismo, deteriorando la imagen proyectada del destino. De igual manera, Bloomberg, Hess y Weerapana (2004) desde la perspectiva económica, indican que no se debe analizar el turismo y el riesgo del destino como dos variables independientes, sino como una relación inversamente proporcional. Así, el proceso de toma de decisiones de los futuros turistas se basa en un modelo actitudinal que toma en consideración el nivel de percepción de riesgo, por lo que si existen conflictos o peligros de su aparición repentina, la llegada de turistas internacionales tenderá a mermar.

Saha y Yap (2013) consideran que si bien los actos terroristas afectan al turismo receptivo, únicamente repercute en la tasa masiva de llegadas, dando mayor relevancia de impacto a la inestabilidad política derivada de la probabilidad de cambios gubernamentales, tensiones religiosas y étnicas, revoluciones violentas y golpes militares, pues estos acontecimientos evidencian una mayor reducción de la actividad turística. Es decir, países con niveles bajos y medios de probabilidad de cambios de regímenes aumentan la demanda turística. Al fin y al cabo, la inestabilidad política reduce el turismo receptivo en cualquier nivel de terrorismo e incluso comprueban que un alto nivel de inestabilidad política produce mayor efecto negativo en el arribo de turistas que un nivel alto de amenaza terrorista. En cambio, el terrorismo per se tiene un efecto mixto añadido: Cuando el país sufre de inestabilidad política y a su vez existe una potencial amenaza 
terrorista, independientemente de su probabilidad, disminuye el número de turistas, mientras que un país con bajo o medio nivel de inestabilidad política, el número de turistas aumenta, siempre y cuando la amenaza de actos terroristas no implique un riesgo político, es decir, que no condicionen la situación política actual de un país (Saha y Yap 2013).

En suma, la inestabilidad política y de seguridad, aunada al factor riesgo de la actividad turística, afecta los recursos psicosociales y la complejidad de la imagen-destino como cuestión perceptiva del prospectivo turista, por lo que en la relación inconsciente costo-beneficio de los procesos de toma de decisiones de los potenciales visitantes extranjeros, la seguridad personal se desmerece transversalmente en cuatro situaciones: a) el terrorismo, b) la guerra y la inestabilidad política, c) las preocupaciones por la salud y, d) la criminalidad (Castaño 2005:108-129), mientras Araña y León (2008) sumaban dos causas a lo anterior: e) la experiencia personal $y, f$ ) los aspectos culturales del destino.

De esta manera se parte de la convicción de que el potencial turista realiza un análisis introspectivo de las relaciones costo-beneficio en la decisión por el destino a visitar, en el que si bien se configuran aspectos como precios, competencias, actividades, historia y cultura, accesibilidad, tiempo de visita, temporadas y necesidades particulares del viaje, existen también limitaciones en la selección del mismo, atribuidas principalmente a la percepción de seguridad personal.

Esta dicotomía determina que el efecto de los ataques terroristas dependerá principalmente del status quo prominente de cada país. En otras palabras, va depender del modelo estructurado con las siguientes variables: país, tiempo, demanda turística del país, producto interno bruto, estabilidad política, terrorismo, tasa de cambio e indicadores.

\section{Percepciones y medios de comunicación}

Los medios de comunicación y más recientemente las redes sociales se constituyen como consignatarias y creadoras de realidades sociales compartidas a través del discurso (Jacoby 1977; Watzlawick 1979; Dominguez 2009), por to que el lenguaje, la cobertura y los tonos de las interacciones sobre los acontecimientos de países lejanos son fundamentales para comprender la migración de preferencias de los destinos y la percepción social de riesgo (Seabra, Abrantes y Lages 2007; Korstanje 2012). Asimismo, las noticias provenientes de agencias y medios de comunicación internacionales influencian las percepciones y actitudes de las audiencias hacia el país al que se refieren, sobre todo porque la mayoría de las personas no tienen acceso a la pluralidad informativa de los eventos internacionales (Wu 1998; López-García 2006). Por tanto, la imagen expuesta en los diferentes canales de transmisión comunicativa sobre destinos en conflicto influencia el componente afectivo de los turistas, afectando la caracterización del evento según las emociones y sentimientos asociados con el país al que se pretende visitar (Hall 2002; Álvarez y Campo 2014).

Es evidente la relación existente entre las percepciones sociales de riesgo y las preocupaciones de seguridad de los turistas. Entre los más comunes se encuentran los efectos del terrorismo en las decisiones de viaje (Pizam y Fleischer 2002; Ritchie 2004; Coshall 2005; Bonham 2006), el impacto del riesgo y la seguridad en el comportamiento del prospectivo turista (Sönmez y Graefe 1998; Reisinger y Mavondo 2006; Irvine y Anderson 2006; Kozak, Crotts y Law 2007; Hunter-Jones, Jeffs y Smith 2008; Lepp y Gibson 2008) y la 
percepción de seguridad y los elementos de riesgo en la toma de decisiones (Lennon, Weber y Henson 2001; Barker, Page y Meyer 2003; Lepp y Gibson 2003; Reisinger y Crotts 2009).

Por su parte, es importante destacar el actual rol que asumen las agencias internacionales de noticias y las cadenas transnacionales en el oligopolio informativo de asuntos extrafronterizos. La mediamorfosis por la que atraviesan los medios de comunicación convencionales (prensa, radio y televisión), compiten en el ecosistema informativo con las redes sociales y los medios digitales (Romero-Rodríguez, Gadea y Hernández Díaz 2015). Por ello, la situación ha obligado a los medios de comunicación nacionales a optar por reducir costos de producción informativa a entre los que se encuentra la eliminación paulatina de corresponsalías fijas en el extranjero (Aguaded y Romero-Rodríguez 2015) ergo mayor dependencia de las cadenas internacionales de noticias-CNN, BBC, Al Jazeera, etc- y agencias internacionales -Reuters, AP, AFP, EFE, etc.-; lo que origina de alguna manera un «efecto ventrílocuo» en el que una sola o pocas perspectivas informativas se asumen como ciertas en todos los medios de comunicación por la propia incapacidad que tiene el sistema mediático de verificar los hechos con personal propio en el terreno (Arráez 1998). En pocas palabras, son estas agencias y medios transnacionales los que fijan la agenda informativa, el enfoque y sus versiones y generan las matrices de opinión de cualquier acontecimiento, aunque en apariencia exista pluralidad producto de la paráfrasis de las mismas en cada medio doméstico o en las propias informaciones que se comparten a través de los nodos de las redes sociales (Romero-Rodríguez 2014).

Según Gurevitch, Levy y Roeh (1991) y Clausen (2004), la cobertura de las noticias internacionales viene enmarcada por una "domesticación" de las informaciones, en el sentido que las agencias y medios internacionales mantienen orientaciones culturales específicas intentando enmarcar (framing), construir -y juzgar- los acontecimientos desde el discurso imperante de sus audiencias, generalmente desde la mirada occidental, donde el respeto por los Derechos Humanos, la democracia, el Estado de Derecho y la institucionalidad, son factores determinantes en la creación del discurso.

Aunque es evidente la preocupación académica acerca del impacto del terrorismo en el turismo y su correlacional efecto económico, no se evidencia un abordaje académico suficiente respecto a la interrelación entre terrorismo, estabilidad política, cobertura de la información internacional y sus efectos en la toma de decisión de viaje desde la óptica de los medios de comunicación como constructores de realidades sociales compartidas. Por ello, el presente estudio asume que la cobertura mediática es mayor cuando los turistas son las víctimas de los ataques terroristas (Brunt, Mawby y Hambly 2000; Maguire, Weatherby y Mathers 2002; Clayton, Mustelier y Maximiliano 2014; Shakeela y Weaver 2016).

Asimismo, cuando los turistas están mejor informados sobre los eventos políticos en la región y comprenden las razones de los ataques terroristas, se consideran menos amenazados por los efectos de los actos terroristas (Hannam, Butler y Paris 2014; Williams y Baláž 2015; Horner y Swarbrooke 2016), mientras que la persistencia de los actos terroristas afecta los elementos perceptivos de reputación del destino como país seguro (Adessky y Freedman 2005; Moten 2010; Van Engeland y Rudolph 2016).

Bajo este planteamiento, se busca realizar un estudio comparativo respecto a la estabilidad política y atentados terroristas expuestos en los medios de comunicación acontecidos en Egipto, España, Túnez y Venezuela para determinar su influencia en el turismo preceptivo. 


\section{Materiales y métodos}

La presente investigación tiene como objetivo general determinar la interrelación entre el enfoque de los medios y agencias internacionales con respecto a la llegada de turistas internacionales. Para ello es necesario cumplir con los siguientes objetivos específicos: (1) Revisar los datos sobre gobernanza, número y efectos de los atentados terroristas y llegadas de turistas internacionales a los citados países en el intervalo de estudio; (2) Analizar cualitativamente la cobertura informativa sobre atentados terroristas y estabilidad política en Túnez, Egipto, España y Venezuela en el período 2012-2015 y; (3) Verificar el cumplimiento de las reglas de Saha y Yap (2013) acerca de que los atentados terroristas no inciden negativamente en la llegada de turistas, siempre y cuando exista estabilidad política.

Para el cumplimiento del primer objetivo específico, meramente descriptivo, se revisarán los indicadores de gobernanza mundial del Banco Mundial en el intervalo 2012-2015. Los números y efectos de los atentados terroristas se obtendrán a través de la revisión de los datos 2012-2015 de los anuarios del Instituto Europeo del Mediterráneo (IEMED 2016) y se relacionarán con las llegadas de turistas internacionales a Egipto, Túnez, España y Venezuela, contabilizadas a través de los datos emitidos por la Organización Mundial del Turismo (UNWTO 2015) en su anuario de estadísticas de turismo 2012-2015, confirmado con las estadísticas oficiales de cada país.

Para el segundo objetivo se aplicó un análisis del discurso de base interpretativa de los titulares de medios y agencias internacionales, con el fin de determinar la proyección informativa sobre los atentados terroristas y la estabilidad política de los cuatro países. Este tipo de método se enfoca hacia descripciones que profundizan en los datos extraídos para desarrollar categorías conceptuales ilustrando de ese modo los presupuestos teóricos del estudio con el propósito de teorizar sobre el fenómeno (Serrano, 1998). Asimismo "implican la interpretación de los hallazgos a través de los estudios del lenguaje para generar nuevas comprensiones inductivas de los fenómenos, eventos o experiencias" (Saini y Shlonsky 2012: 31). Se realizará una selección de los 4 medios de comunicación y las 4 agencias internacionales de noticias con mayor tráfico web ${ }^{1}$ en base al Ranking Alexa, ya que se entiende que para el proceso de las coberturas informativas domésticas, los profesionales de la información acceden a ellas por esta vía.

Tabla 1: Agencias y medios de comunicación internacionales con mayor tráfico Web

\begin{tabular}{|cccc|}
\hline $\begin{array}{c}\text { Agencia } \\
\text { internacional }\end{array}$ & $\begin{array}{c}\text { Lugar en el } \\
\text { ranking }\end{array}$ & $\begin{array}{c}\text { Medio de } \\
\text { comunicación }\end{array}$ & $\begin{array}{c}\text { Lugar en el } \\
\text { ranking }\end{array}$ \\
\hline Reuters & 377 & MSN & 26 \\
\hline $\begin{array}{c}\text { Russia Today } \\
\text { (RT) }\end{array}$ & 418 & BBC & 82 \\
\hline Al Jazeera & 1.451 & CNN & 88 \\
\hline Associated & 2188 & New York & 112 \\
\hline
\end{tabular}

\footnotetext{
${ }^{1}$ Ranking Alexa Analytics. http://www.alexa.com/topsites
} 


Press (AP) Times

Fuente: elaboración propia a partir de los datos de Alexa Analytics Toolkit

Seleccionados los medios y las agencias, se procederá a realizar una criba de aquellos titulares que contienen información sobre los países objeto del presente estudio en el período 2012-2015, que contengan códigos correspondientes a estabilidad política, social y económica, conflictos y atentados terroristas, para realizar el análisis de contenido a través del software MAXQDA (v. 11.0.11), con el fin de clasificar las unidades hermenéuticas, códigos, familias y árboles de relación semiótica y así extraer la proyección discursiva de los cuatro países a través de los medios y agencias estudiadas.

En relación al tercer y último objetivo específico, serán contrastados comparativamente los resultados obtenidos a partir de los estudios referidos ut supra con respecto a las conclusiones de Saha y Yap (2013). Para ello se realizará una discusión de los resultados tangibles en función de verificar la incidencia o no de los atentados terroristas con respecto a la llegada de turistas internacionales en los cuatro países. En otras palabras, se estableció una conexión de los resultados extraídos del análisis cuantitativo de llegadas de turistas y del estudio de contenido de las agencias y medios internacionales para verificar la correlación estabilidad/atentados, en el cumplimiento de las reglas propuestas por Saha y Yap (2013) acerca de la incidencia del terrorismo y la estabilidad política en el turismo.

Como hipótesis de partida $(\mathrm{H} 0)$ se cree que las informaciones de las agencias y medios internacionales son determinantes en la creación de percepciones globales sobre los países en análisis, por lo que (H1) las llegadas de turismo internacional a cada país serán inversamente proporcionales a la tensión o percepción de riesgo que muestre la información internacional, $(\mathrm{H} 2)$ cumpliéndose las conclusiones planteadas por Saha y Yap (2013) sobre la ausencia de incidencia del terrorismo en la llegada de turistas internacionales.

En la muestra se analizarán los datos de Egipto, Túnez, España y Venezuela, según los datos del Banco Mundial (2015). En este sentido, la muestra permitirá revisar un país con alto número de atentados terroristas y poco índice de gobernabilidad (Egipto), uno con índice intermedio de gobernabilidad y alto número de atentados terroristas (Túnez), uno sin atentados terroristas pero con bajo índice de gobernabilidad (Venezuela) y por último, uno con mínimo valor de atentados terroristas y con un buen índice de gobernabilidad (España).

Para el cumplimiento de los objetivos específicos, se utilizará un diseño cuantitativo a través de datos cruzados de llegadas de turistas internacionales a Egipto, Túnez, España y Venezuela entre 2012 y 2015 provenientes de organizaciones internacionales y europeas competentes en la materia. Posteriormente, y para hacer frente al segundo objetivo específico, será realizado un análisis de contenido de base interpretativa de los titulares de las agencias y medios de comunicación internacionales que guarden relación con los cuatro países escogidos para el período de estudio. El tipo de estudio interpretativo involucra el entrecruzamiento de resultados con el fin de generar la comprensión inductiva de fenómenos, eventos y experiencias (Saini y Shlonsky 2012). Esta fase permitirá revisar empíricamente la interrelación entre la proyección imaginaria del destino y el comportamiento del prospecto consumidor turístico. 


\section{Resultados}

\section{Estabilidad política}

Según Hurwitz (1973) las percepciones pueden trasladarse a variables cuantificables. De esta forma se entiende que la estabilidad política está conformada por: a) La ausencia de violencia o del ejercicio de la misma; b) la duración del gobierno; c) La existencia de un régimen constitucional; d) La ausencia de cambios estructurales y; e) Los atributos societarios.

El Banco Mundial (2016) realiza todos los años un estudio cuantitativo de la estabilidad política de cada país, tomando en consideración las dimensiones en referencia, pero también vinculándolas con la actividad terrorista, los conflictos religiosos, étnicos, sociales y la polarización de sus sociedades. El indicador, cuyos valores se contabilizan de 0 (menor estabilidad) a 100 (mayor estabilidad), intenta mostrar grosso modo las variaciones anuales de las dimensiones ut supra mencionadas, englobándolas en una ponderación promediada.

Para el cumplimiento de nuestro primer objetivo específico, se procedió a obtener dichos valores promedios en el rango 2012-2015 de los países objeto de estudio, con el fin de obtener descriptivamente la situación valorativa de cada uno de los destinos.

Gráfico 1: Indicadores de estabilidad política y ausencia de violencia 2012-2015 de Egipto, Túnez, España y Venezuela

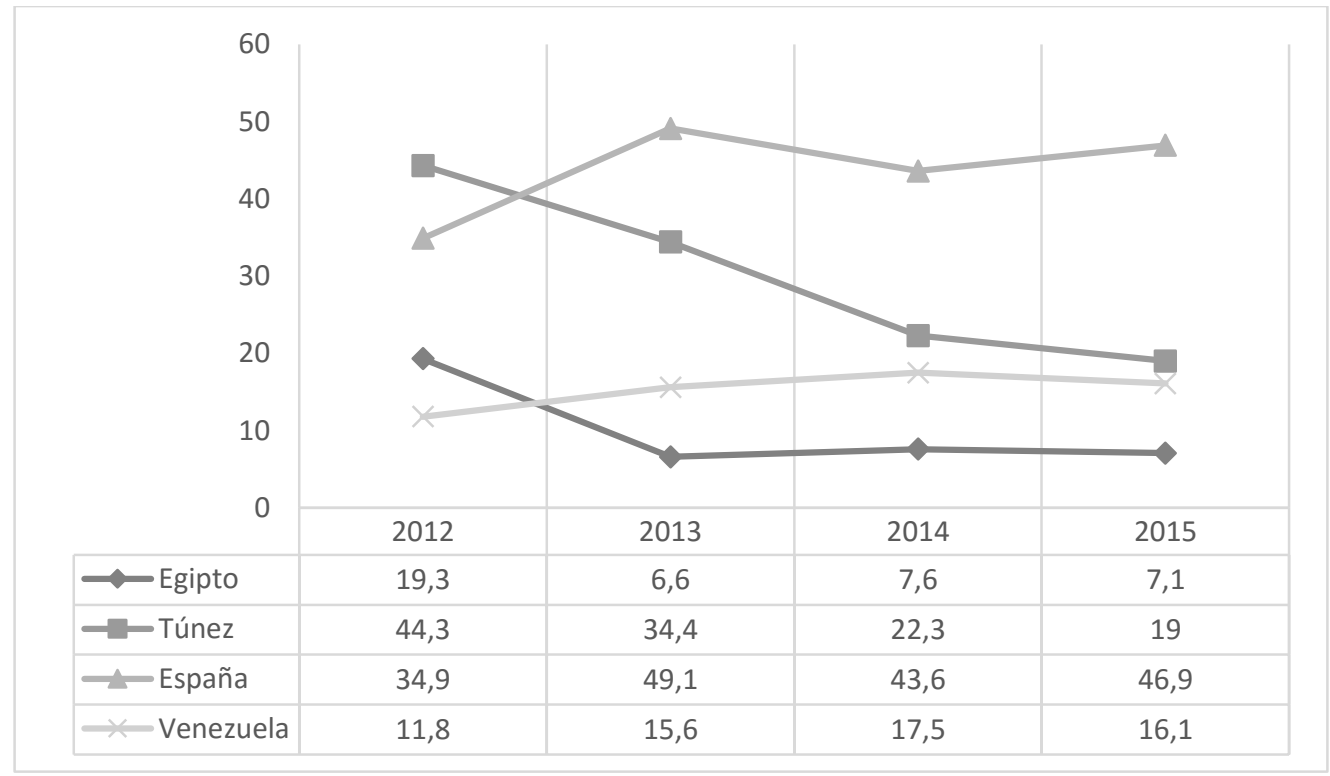

Fuente: elaboración propia a partir de los datos del Worldwide Governance Indicators. Banco Mundial.

El gráfico anterior refleja con meridiana claridad que España, con un promedio de 43,62\% de estabilidad política y ausencia de violencia, lidera el valor positivo de los países en análisis, mientras que Egipto, por el contrario, representa un riesgo latente de estabilidad con un promedio de 10,15, más de 33 puntos de diferencia. Túnez promedia con una puntuación de 30,0 y Venezuela se ubica en segundo lugar con mayor 
riesgo de inestabilidad con 15,25, según las cifras extraídas del índice de gobernanza mundial del Banco Mundial.

Los picos descendentes que se manifiestan en el gráfico coinciden con tensiones internas, conmoción interior o atentados terroristas. Así, Túnez en el 2012 obtenía 44,3 puntos y bajaba 10 puntos en 2013, año en que se consolidaron cambios gubernamentales que originaron posteriormente una transición a la democracia marcada por el asesinato de líderes políticos, atentados terroristas y la violencia islamista con los salafistas y la Liga para la Protección de la Revolución.

Tabla 2: Número de atentados terroristas y muertes por atentado 2012-2015 en Egipto y Túnez

\begin{tabular}{|c|c|c|c|c|c|c|c|c|c|c|}
\hline & \multicolumn{5}{|c|}{ EGI } & \multicolumn{5}{|c|}{ TUN } \\
\hline & 2012 & 2013 & 2014 & 2015 & Total & 2012 & 2013 & 2014 & 2015 & Total \\
\hline Atentados & 2 & 13 & 10 & 36 & 61 & 0 & 3 & 2 & 4 & 9 \\
\hline $\begin{array}{c}\text { Muertes por } \\
\text { atentado }\end{array}$ & 22 & 231 & 142 & 567 & 962 & 0 & 11 & 2 & 47 & 60 \\
\hline $\begin{array}{l}\text { Promedio } \\
\text { atentados }\end{array}$ & \multicolumn{5}{|c|}{15,25} & \multicolumn{5}{|c|}{2,25} \\
\hline $\begin{array}{l}\text { Promedio } \\
\text { muertes por } \\
\text { atentados }\end{array}$ & \multicolumn{5}{|c|}{240,5} & \multicolumn{5}{|c|}{15} \\
\hline
\end{tabular}

Además de toda la inestabilidad política que surge en el Magreb y Medio Oriente, hace aparición la milicia islamista del Estado Islámico (ISIS), organización terrorista próxima a Al Qaeda que nace en 2003 para hacer frente a la invasión de Irak, pero que reclama la creación de un califato de todo el mundo musulmán y el reclamo de las tierras del Al-Andalus (hoy en día Andalucía, al sur de España).

Con la finalidad de entrever la repercusión que tiene el terrorismo y la inestabilidad política en el turismo receptivo, es necesario revisar las coincidencias existentes entre estas variables, por lo que se desglosa a continuación la comparativa anual en cada uno de los países en análisis.

\footnotetext{
${ }^{2}$ Global Terrorism Index 2014 del Institute for Economic \& Peace, disponible en línea en: http://goo.gl/7shM9E (Consultado el 7/8/2015).
} 
Tabla 3: Número de arribos de turistas extranjeros 2012-2015 en Egipto, Túnez, España y Venezuela

\begin{tabular}{ccccc}
\hline & EGI & TUN & ESP & VEN \\
\hline $\mathbf{2 0 1 2}$ & 14.730 .813 & 6.902 .749 & 52.676 .972 & 535.270 \\
\hline $\mathbf{2 0 1 3}$ & 9.845 .066 & 4.785 .119 & 56.176 .866 & 625.224 \\
\hline $\mathbf{2 0 1 4}$ & 11.531 .858 & 5.950 .464 & 57.464 .496 & 1.061 .020 \\
\hline $\mathbf{2 0 1 5}$ & 9.464 .349 & 6.268 .582 & 60.661 .073 & 1.084 .776
\end{tabular}

Fuentes: elaboración propia a partir de los datos de los anuarios del Instituto Europeo del Mediterráneo (IEMED) y del World Turism Organization (UNWTO)

\section{Proyección informativa}

En relación a la proyección informativa de los destinos turísticos en análisis, fueron extraídos un total de 2100 unidades informativas de los 4 destinos, provenientes del período 2012-2015 de las cuatro agencias internacionales de noticias (Reuters, Russia Today, Al Jazeera y Associated Press) y de los cuatro medios de comunicación internacionales (MSN, BBC, CNN y New York Times). De este total, 568 pertenecen a informaciones sobre Egipto, 460 sobre Túnez, 618 sobre España y 454 sobre Venezuela.

Para el análisis cuanti-cualitativo de todas las unidades informativas recogidas, fue necesario preclasificar los códigos emergentes en familias hermenéuticas y árboles de relación de la siguiente manera: 1) Familia percepción positiva del destino, que incluye los códigos relativos a: 1.1) Ausencia de conflictos y atentados terroristas; 1.2) Estabilidad económica; 1.3) Estabilidad social, contentiva de: 1.3.1) Respeto a los Derechos Humanos; 1.3.2) Seguridad ciudadana; 1.3.3) Coexistencia étnica o social; 1.3.4) Coexistencia religiosa; y; 1.4) Estabilidad política, clasificada en: 1.4.1) Estabilidad gubernamental; 1.4.2) Gobernabilidad y; 1.4.3) Otros aspectos de estabilidad política. En contraparte, los códigos emergentes de 2) Familia percepción negativa del destino, se clasificaron en: 2.1) Conflictos y atentados terroristas; 2.2) Inestabilidad económica; 2.3) Inestabilidad social, contentiva de: 2.3.1) Falta de respeto a los Derechos Humanos; 2.3.2) Delincuencia y tráfico ilegal; 2.3.3) Conflictos étnicos y/o sociales; 2.3.4) Conflictos religiosos; 2.3.5) Otros aspectos de inestabilidad social y; 2.4) Inestabilidad política, conformada por: 2.4.1) Golpes de Estado; 2.4.2) Cambios gubernamentales o de gobierno y; 2.4.3) Otros aspectos de inestabilidad política.

Para la cuantificación de la proyección informativa de los destinos, fue desarrollada una clasificación de las 2100 unidades informativas emergentes en función de su naturaleza dentro de los códigos preclasificados ut supra. Aquellas informaciones no relacionadas con los aspectos referidos (como información deportiva, científica o neutral), no fueron tomadas en consideración en el presente análisis.

En lo relativo a la familia de códigos "Percepción positiva", emergieron un total de 503 unidades informativas, lo que significa un $23,95 \%$ de la totalidad de informaciones extraídas de los medios y agencias, clasificándose de la siguiente manera: 
Tabla 4: Cuantificación de códigos emergentes por destino en la familia "Percepción positiva"

\begin{tabular}{|c|c|c|c|c|c|c|}
\hline Familia & Código emergente & Sub-códigos emergentes & $E G I$ & TUN & $E S P$ & VEN \\
\hline \multirow{9}{*}{ 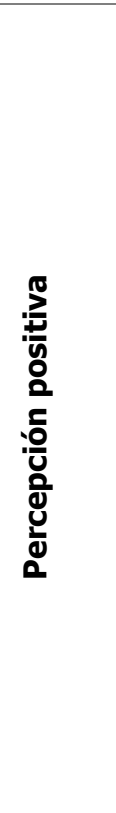 } & $\begin{array}{c}\text { Ausencia de conflictos y } \\
\text { atentados }\end{array}$ & & 3 & 6 & 11 & 0 \\
\hline & Estabilidad económica & & 8 & 22 & 45 & 18 \\
\hline & \multirow{4}{*}{ Estabilidad Social } & $\begin{array}{c}\text { Respeto a los Derechos } \\
\text { Humanos }\end{array}$ & 4 & 7 & 41 & 9 \\
\hline & & Seguridad Ciudadana & 9 & 2 & 74 & 12 \\
\hline & & Coexistencia étnica o social & 2 & 3 & 11 & 2 \\
\hline & & Coexistencia religiosa & 3 & 2 & 11 & 0 \\
\hline & \multirow{3}{*}{ Estabilidad política } & Estabilidad Gubernamental & 0 & 5 & 11 & 40 \\
\hline & & Gobernabilidad & 8 & 36 & 55 & 14 \\
\hline & & Otros estabilidad política & 1 & 11 & 5 & 12 \\
\hline \multicolumn{3}{|c|}{ Total percepción positiva } & 38 & 94 & 264 & 107 \\
\hline
\end{tabular}

Fuente: elaboración propia

En el caso contrario, los códigos emergentes clasificados en el árbol de relación "Percepción negativa", contabilizaron el restante de 1597 unidades informativas, relativas al 76,05\% de las informaciones extraídas, quedando de la siguiente manera:

Tabla 5: Cuantificación de códigos emergentes por destino en la familia «Percepción negativa»

\begin{tabular}{|c|c|c|c|c|c|c|}
\hline Familia & Código emergente & Sub-códigos emergentes & $E G I$ & TUN & $E S P$ & VEN \\
\hline \multirow{6}{*}{ 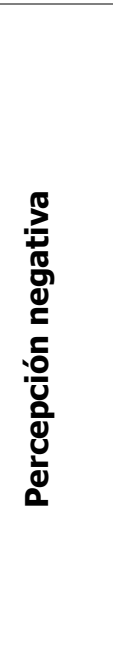 } & $\begin{array}{c}\text { Conflictos y atentados } \\
\text { terroristas }\end{array}$ & & 63 & 30 & 5 & 9 \\
\hline & $\begin{array}{l}\text { Inestabilidad } \\
\text { económica }\end{array}$ & & 16 & 27 & 129 & 90 \\
\hline & \multirow{4}{*}{ Inestabilidad Social } & $\begin{array}{l}\text { Falta de respeto a los Derechos } \\
\text { Humanos }\end{array}$ & 62 & 51 & 28 & 53 \\
\hline & & Delincuencia / Tráfico ilegal & 4 & 6 & 24 & 54 \\
\hline & & Conflictos étnicos o sociales & 49 & 35 & 52 & 21 \\
\hline & & Conflictos religiosos & 61 & 59 & 1 & 1 \\
\hline
\end{tabular}




\begin{tabular}{cccccc}
\hline & Otros inestabilidad social & 4 & 7 & 19 & 2 \\
\cline { 2 - 5 } Inestabilidad política & Cambios gubernamentales & 187 & 69 & 18 & 36 \\
\cline { 2 - 6 } & Golpes de Estado & 68 & 7 & 0 & 33 \\
\cline { 2 - 6 } & Otros inestabilidad política & 28 & 67 & 65 & 52 \\
\hline Total percepción negativa & 542 & 358 & 341 & 351 \\
\hline
\end{tabular}

Fuente: elaboración propia

A fin de tener una mayor claridad sobre la proyección de los destinos en análisis, se procede a realizar una operación de sustracción de valores de percepción positiva a aquellos emergentes en la familia de percepción negativa, con el fin de obtener el coeficiente informativo total de cada país en el período 2012-2015:

Tabla 6: Totalización cuanti-cualitativa de códigos emergentes por destino

\begin{tabular}{|c|c|c|c|c|c|c|}
\hline Familia & Código emergente & Sub-códigos emergentes & $E G I$ & TUN & $E S P$ & VEN \\
\hline \multirow{10}{*}{ 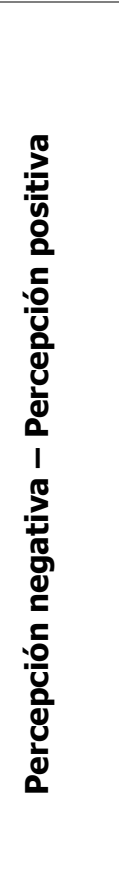 } & $\begin{array}{c}\text { Conflictos y atentados } \\
\text { terroristas }\end{array}$ & & -60 & -24 & +6 & -9 \\
\hline & Estabilidad económica & & -8 & -5 & -84 & -72 \\
\hline & \multirow{5}{*}{ Estabilidad Social } & $\begin{array}{c}\text { Respeto a los Derechos } \\
\text { Humanos }\end{array}$ & -58 & -44 & +13 & -44 \\
\hline & & Seguridad Ciudadana & 5 & -4 & +50 & -42 \\
\hline & & Coexistencia étnica o social & -47 & -32 & -41 & -19 \\
\hline & & Coexistencia religiosa & -58 & -57 & +10 & -1 \\
\hline & & Otros estabilidad social & -4 & -7 & -19 & -2 \\
\hline & \multirow{3}{*}{ Estabilidad política } & Estabilidad gubernamental & -187 & -64 & -7 & +4 \\
\hline & & Gobernabilidad & -60 & +29 & +55 & -19 \\
\hline & & Otros estabilidad política & -27 & -56 & -60 & -40 \\
\hline \multicolumn{3}{|c|}{ Total percepción } & -504 & -264 & -77 & - \\
\hline
\end{tabular}

Nota: En aquellas que aparece el signo (-) significa que es mayor su percepción negativa que positiva en los medios y agencias estudiados. Por el contrario, en aquellas que aparece el signo (+), significa que es mayor su percepción positiva. El sub-código emergente "otros inestabilidad social" solo estuvo presente en la familia de códigos de percepción negativa.

Como se puede observar, Egipto, que totalizaba 580 unidades informativas, queda en primer lugar en la percepción negativa con 504 informaciones negativas (86,89\%); le sigue Túnez con 264 impactos negativos 
de un total de 452 unidades informacionales, lo que reporta $58,40 \%$ de discurso negativo del total de la muestra analizada de ese país; muy cerca se encuentra Venezuela con 244 códigos emergentes de percepción negativa de un total de 458 informaciones extraídas, que representan el 53,27\% de representación negativa. Por último España, con un total de 605 códigos emergentes, presenta -77 puntos negativos en la diferencia cuantitativa de las informaciones.

Tabla 7: Comparativa anual 2012-2015 entre estabilidad política, número de atentados, muertes en atentados y turismo receptivo

\begin{tabular}{|c|c|c|c|c|c|}
\hline & & 2012 & 2013 & 2014 & 2015 \\
\hline \multirow[t]{4}{*}{ Egipto } & Estabilidad política & 19,3 & 6,6 & 7,6 & 7,1 \\
\hline & Número de atentados & 2 & 13 & 10 & 36 \\
\hline & Muertes en atentados & 22 & 231 & 142 & 567 \\
\hline & Número de turistas extranjeros & 14.730 .813 & 9.845 .066 & 11.531 .858 & 9.464 .349 \\
\hline \multirow[t]{4}{*}{ Túnez } & Estabilidad política & 44,3 & 34,4 & 22,3 & 19 \\
\hline & Número de atentados & 0 & 3 & 2 & 4 \\
\hline & Muertes en atentados & 0 & 11 & 2 & 47 \\
\hline & Número de turistas extranjeros & 6.902 .749 & 4.785 .119 & 5.950 .464 & 6.268 .582 \\
\hline \multirow[t]{4}{*}{ España } & Estabilidad política & 34,9 & 49,1 & 43,6 & 46,9 \\
\hline & Número de atentados & 0 & 0 & 0 & 0 \\
\hline & Muertes en atentados & 0 & 0 & 0 & 0 \\
\hline & Número de turistas extranjeros & 52.676 .972 & 56.176 .866 & 57.464 .496 & 60.661 .073 \\
\hline \multirow[t]{4}{*}{ Venezuela } & Estabilidad política & 11,8 & 15,6 & 17,5 & 16,1 \\
\hline & Número de atentados & 0 & 0 & 0 & 0 \\
\hline & Muertes en atentados & 0 & 0 & 0 & 0 \\
\hline & Número de turistas extranjeros & 535.270 & 625.224 & 1.061 .020 & 1.084 .776 \\
\hline
\end{tabular}

Fuente: elaboración propia

El análisis del discurso mediático por códigos emergentes permite distinguir con meridiana claridad que la inestabilidad gubernamental, la ausencia de gobernabilidad, la falta de respeto a los Derechos Humanos y la posibilidad de conflictos y/o atentados terroristas, son los aspectos negativos más impactantes de Egipto como destino turístico. Asimismo, la inestabilidad política, los conflictos religiosos y la probabilidad de atentados terroristas forman parte de la realidad discursivo-mediática de Túnez. 
Por su parte, Venezuela es referida en el extracto informativo como un país con poca estabilidad económica, potenciales problemas de inestabilidad política y de seguridad ciudadana, así como conculcador de los derechos fundamentales; mientras que España basa su percepción negativa en la inestabilidad económica.

\section{Discusiones y conclusión}

Respecto a la primera hipótesis ( $\mathrm{H} 1)$, los datos ofrecidos por el Worldwide Governance Indicators del Banco Mundial sobre estabilidad política y ausencia de violencia 2012-2015 son similares a la información extraída de las agencias y medios internacionales en la creación de percepciones globales de los países analizados. Por una parte, España y Egipto representan los extremos en torno a los factores determinantes de la estabilidad política, el primero por su incuestionable presencia y el siguiente por su incuestionable ausencia. En cambio, en el caso de Venezuela y Túnez, si bien contienen factores que fluctúan cuando se comparan las percepciones de las agencias y medios internacionales con los indicadores de estabilidad política del Banco Mundial, la franja de estabilidad política que ocupan duplica el nivel de Egipto, pero sin aproximarse a las cifras óptimas de España.

En referencia a la segunda hipótesis (H2), Sonmez y Graefe (1998) y Bloomberg, Hess y Weerapana (2002) avalan que el turismo receptivo es una variable inversamente proporcional a la percepción de riesgo producido por los conflictos y atentados terroristas. Tomando en cuenta la percepción de los medios y agencias internacionales respecto al riesgo de una acción repentina en el país de destino entre 2012-2015, se evidencian las siguientes circunstancias: En España, con una percepción nula de ataques y con acciones para asegurar la tranquilidad de los ciudadanos frente a la amenaza terrorista (+6), el turismo ha aumentado en un 15\% (7.984.101). En Egipto con una amenaza factible de ataques provenientes del Estado Islámico (-60), el turismo ha disminuido en un 35\% (5.266.464). Túnez, muy próximo a las cifras de Egipto, al estar inmerso en una coyuntura similar (-44), refleja la caída del turismo en un 9\% (634.167). Por último, Venezuela, posee una percepción negativa mínima de conflicto y amenaza terrorista en comparación con los países norafricanos mencionados, causada por las suposiciones hacia una posible intervención y ataque estadounidense en el país (-9), a pesar de ello, el turismo ha aumentado en $102 \%$ (549.506).

En los cuatro casos queda patente la incidencia de la percepción/riesgo de terrorismo en el turismo. Cuando un país es proclive a recibir atentados, los medios y agencias de comunicación se hacen eco de la situación, siendo determinantes en la llegada de turistas al destino. En este sentido, esta investigación valida que la llegada de turistas es inversamente proporcional a la percepción que exista de los conflictos y atentados terroristas. A partir de esa verificación, se puede profundizar en el análisis del mejoramiento de imagen hacia el turismo de aquellos países que han sido víctimas de atentados terroristas y en efecto toma mayor valor la perspectiva académica que asume que los atentados terroristas buscan afectar el ingreso económico a través de la disminución del turismo receptivo.

Para finalizar, en la tercera hipótesis (H3) se busca comprobar la percepción de estabilidad del destino en relación con la llegada de turistas. La teoría de Saha y Yap (2013) estima que la estabilidad política tiene mayor influencia en la llegada de turistas que la amenaza de atentados. Continuando con la percepción de los medios y agencias de comunicación internacionales, destaca que en España existe una percepción menos negativa que los demás países (-77), producida primordialmente por la gobernabilidad $(+55)$ y otros 
elementos menos comunes que constituyen la estabilidad política (+60), como es el caso del desarrollo de energías renovables, además de elementos de estabilidad social como la seguridad ciudadana $(+50)$ y respeto a los derechos humanos (+13). En el caso de Egipto se presenta una percepción negativa totalizada (-504) generada por factores de estabilidad política. Entre los más determinantes se encuentran la estabilidad gubernamental (-187) y gobernabilidad (-60), además de la coexistencia étnica y social (-58), como factores de estabilidad social con mayor agravio. En los casos de Túnez (-264) y Venezuela (-244), se observa una particularidad, aunque de ambos países se tiene una percepción negativa, en Túnez disminuye el turismo, pero en Venezuela se duplica en las fechas de análisis (2012-2015). Independientemente de si la percepción es menos negativa en Túnez respecto a la estabilidad económica y la seguridad ciudadana, mientras que en Venezuela la percepción es menos negativa en la coexistencia religiosa y en la estabilidad gubernamental, la tesis propugnada por Saha y Yap (2013) no se manifiesta con exactitud, ya que en el análisis cuantitativo así como en el análisis del discurso periodístico se verifica que el terrorismo tiene mayor trascendencia que la inestabilidad política y económica.

Conviene a modo de reflexión, determinar con mayor detalle en futuras investigaciones los efectos del discurso bélico de agencias y medios internacionales (Torres-Toukoumidis, et al. 2017), especialmente profundizando en otras variables que pudiera afectar la percepción sociopolítica del destino expuesto desde los medios y agencias internacionales, contrastando a su vez con la exposición de los acontecimientos en los medios nacionales. Complementariamente, valdría incorporar el análisis sobre la existencia de publicidad de las actividades turísticas dentro del ecosistema mediático internacional con la finalidad de evaluar la intervención de los gobiernos respecto a la imagen que buscan exponer sobre sus países.

\section{Referencias}

Adessky, R. \& Freedman, S. (2005). Treating survivors of terrorism while adversity continues. Journal of aggression, maltreatment \& trauma, 10 (2), 443-454. doi: https://doi.org/10.1300/j146v10n01 03

Aguaded, I. \& Romero-Rodríguez, L. (2015). Mediamorfosis y desinformación en la infoesfera: Alfabetización mediática, digital e informacional ante los cambios de hábitos de consumo informativo. Education in the Knowledge Society, 16 (1), 44-57. https://doi.org/10.14201/eks20151614457

Álvarez, M. \& Campo, S. (2014). The influence of political conflicts on country image and intention to visit: A study of Israel's image. Tourism Management, 40, 70-78. https://doi.org/10.1016/j.tourman.2013.05.009

Araña, J. \& León, C. (2008). The Impact of Terrorism on Tourism Demand. Annals of Tourism Research, 35, 299-215. https://doi.org/10.1016/j.annals.2007.08.003

Arráez, R. (1998). La comunicación para el desarrollo. Su aplicación para el medio radio. Revista Latina de Comunicación Social, 9 (1).

Banco Mundial. (2015). El índice de gobernabilidad se extrae de los indicadores del "Worldwide Governance Indicators. http://info.worldbank.org/governance/wgi/index.aspx\#home

Barker, M., Page, S. \& Meyer, D. (2003). Urban visitor perceptions of safety during a special event. Journal of Travel Research, 41 (4), 355-361. https://doi.org/10.1177/0047287503041004004 
Blomberg, S., Hess, G. \& Weerapana, A. (2004). Economic Conditions and Terrorism. European Journal of Political Economy, 20 (2), 463-78.

Bonham, C. (2006). The impact of 9/11 and other terrible global events on tourism in the United States and Hawaii. Journal of Travel Research, 45(1), 99-110. https://doi.org/10.1177/0047287506288812

Brunt, P., Mawby, R. \& Hambly, Z. (2000). Tourist victimisation and the fear of crime on holiday. Tourism Management, 21 (4), 417-424. doi: https://doi.org/10.1016/s0261-5177(99)00084-9

Castaño, J. (2005). Psicología social de los viajes y del turismo. Madrid: Thomson.

Clausen, L. (2004). Localizing the global: Domestication'processes in international news production. Media, Culture \& Society, 26 (1), 25-44. https://doi.org/10.1177/01634433704038203

Clayton, A., Cisneros, L, \& Korstanje, M. (2014). Understanding perceptions and attitudes to Risk in the Tourism Industry. International Journal of Religious Tourism and Pilgrimage, 2 (8), 48-57. Recuperado de http://arrow.dit.ie/cqi/viewcontent.cqi?article=1051\&context=ijitp

Coshall, J. (2005). Interventions on UK earnings and expenditures overseas. Annals of Tourism Research, 32 (3), 592-609. https://doi.org/10.1016/j.annals.2004.09.004

Domínguez, D. (2009). Democracia 2.0: La política se introduce en las redes sociales. Pensar la publicidad, $3(2), 31-48$.

Gurevitch, M., Levy, M. \& Roeh, I. (1991). The global newsroom: Convergences and diversities in the globalization of television news. Communication and citizenship: Journalism and the public sphere, 195-216.

Hall, M. (2002). Travel safety, terrorism and the media: the significance of the issue-attention cycle. Current Issues in Tourism, 5 (5), 458-466. https://doi.org/10.1080/13683500208667935

Hannam, K., Butler, G. \& Paris, C. (2014). Developments and key issues in tourism mobilities. Annals of Tourism Research, 44, 171-185.doi: https://doi.org/10.1016/j.annals.2013.09.010

Horner, S. \& Swarbrooke, J. (2016). Consumer behaviour in tourism. Abingdon-on-Thames: Routledge.

Hunter-Jones, P., Jeffs, A. \& Smith, D. (2008). Backpacking your way into crisis: an exploratory study into perceived risk and tourist behaviour amongst young people. Journal of Travel \& Tourism Marketing, 2 (4), 237-247 https://doi.org/10.1300/j073v23n02 18

Hurwitz, L. (1973). Contemporary approaches to political stability, Comparative Politics, 5 (3), 449-463.

IEMED. (2011). Mediterranean Yearbook. Instituto Europeo del Mediterráneo. http://www.iemed.org/publicacions-es/historic-de-publicacions/anuari-de-la-mediterrania/anuaride-la-mediterrania-2011

IEMED. (2016). Mediterranean Yearbook. Instituto Europeo del Mediterráneo. http://www.iemed.org/publicacions-es/historic-de-publicacions/anuari-de-la-mediterrania/anuariiemed-de-la-mediterrania-2016

Institute for Economic \& Peace. (2015). Global Terrorism Index. http://economicsandpeace.org/wpcontent/uploads/2015/11/Global-Terrorism-Index-2015.pdf

Irvine, W. \& Anderson, A. (2006). The effect of disaster on peripheral tourism places and the disaffection of prospective visitors. Tourism, security \& safety, 169-186. https://doi.org/10.1016/b978-0-75067898-8.50016-3

Jacoby, J. (1977). Information Load and Decision Quality: Some Contested Issues. Journal of Marketing Research, 14(4), 569-573. https://doi.org/10.2307/3151201 
Korstanje, M. (2012). ¿ Se puede ponderar la seguridad turística? Un ensayo conceptual. Turismo e Sociedade, 5 (2). https://doi.org/10.5380/tes.v5i2.26332

Kozak, M., Crotts, J. \& Law, R. (2007). The impact of the perception of risk on international travelers. International Journal of Tourism Research, 9 (4), 233-242. http://doi.org/10.1002/jtr.607

Lennon, R., Weber, M. \& Henson, J. (2001). A test of a theoretical model of consumer travel behaviour: German consumers' perception of Northern Ireland as a tourist destination. Journal of Vacation Marketing, 7 (1), 51-62. https://doi.org/10.1177/135676670100700105

Lepp, A. \& Gibson, H. (2008). Sensation seeking and tourism: Tourist role, perception of risk and destination choice. Tourism Management, 29 (4), 740-750. https://doi.org/10.1016/j.tourman.2007.08.002

López García, G. (2006). Comunicación digital y líneas de fractura en el paradigma de la agenda setting.. Doxa. Comunicación 4, 37-58.

Maguire, B., Weatherby, G. \& Mathers, R. (2002). Network news coverage of school shootings. The Social Science Journal, 39 (3), 465-470. https://doi.org/10.1016/s0362-3319(02)00201-x

Moten, A. (2010). Understanding terrorism: Contested concept, conflicting perspectives and shattering consequences. Intellectual Discourse, 18 (1), 35-63. Recuperado de http://journals.iium.edu.my/intdiscourse/index.php/islam/article/download/137/138

Pizam A. \& Fleischer, A. (2002). Severity versus frequency of acts of terrorism: Which has a larger impact on tourism demand?. Journal of Travel Research, 40 (3): 337-339. https://doi.org/10.1177/0047287502040003011

Reinares, F. (2012). Geografía mundial y del terrorismo. Madrid: Real Instituto El Cano.

Reisinger, Y. \& Mavondo, F. (2006). Cultural differences in travel risk perception. Journal of Trave/ \& Tourism Marketing 20 (1), 13-31. https://doi.org/10.1300/j073v20n01 02

Reisinger, Y. \& Crotts, J. (2009). The influence of gender on travel risk perceptions, safety, and travel $\begin{array}{lllll}\text { intentions. } & \text { Tourism } & \text { Analysis, }\end{array}$ https://doi.org/10.3727/108354210x12645141401269

Ritchie, B. (2004). Chaos, crises and disasters: a strategic approach to crisis management in the tourism industry. Tourism Management, 25(6), 669-683. https://doi.org/10.1016/j.tourman.2003.09.004

Romero-Rodríguez, L. (2014). Pragmática de la desinformación: Estratagemas e incidencia de la calidad informativa de los medios, disertación doctoral, Universidad de Huelva.

Romero-Rodríguez, L., Gadea, W. \& Hernández Díaz, G. (2015). Incidencia de la diversificación del ecosistema comunicativo en la sobresaturación informativa. Comunicación. Estudios Venezolanos de la Comunicación, (171-172), 24-33.

Saha, S. \& Yap, G. (2013). The moderation effects of political instability and terrorism on tourism development: a cross-country panel analysis. Journal of Travel Research, 53 (4), 509-521. https://doi.org/10.1177/0047287513496472

Saini, M. \& Shlonsky. A. (2012). Systematic synthesis of qualitative research. England: Oxford University Press.

Seabra, C, Abrantes, J. \& Lages, L. (2007). The impact of using non-media information sources on the future use of mass media information sources: The mediating role of expectations fulfillment. Tourism Management, 28 (6), 1541-1554. https://doi.org/10.1016/j.tourman.2007.02.008

Serrano, G.P. (1998). Investigación cualitativa. Retos e interrogantes. I. Métodos. Madrid: La Muralla. 
Shakeela, A. \& Weaver, D. (2016). The exploratory social-mediatized gaze: Reactions of virtual tourists to an inflammatory YouTube incident. Journal of Travel Research, 55 (1), 113-124. Recuperado de http://journals.sagepub.com/doi/abs/10.1177/0047287514532369

Soengas, X. (2013). El papel de Internet y de las redes sociales en las revueltas árabes: Una alternativa a la censura de la prensa oficial. Comunicar, 4 (21), 147-155. https://doi.orq/10.3916/c41-2013-14

Sönmez, S. (1998). Influence of terrorism risk on foreign tourism decisions. Tourism, Terrorism and political instability. Annals of Tourism Research, 25 (2), 416-456. https://doi.org/10.1016/s01607383(97)00093-5

Sönmez, S. \& Graefe, A. (1998). Determining future travel behavior from past travel experience and perceptions of risk and safety. Journal of Travel Research, 37 (2), 171-177. https://doi.org/10.1177/004728759803700209

Torres-Toukoumidis,A., Romero-Rodríguez,L., De-Casas-Moreno, P. \& Aguaded.I (2017)."Construcción del discurso bélico desde las agencias internacionales de noticias: Estudio de Caso Atentados del 13 de noviembre de 2015. Revista Mediterránea de Comunicación/Mediterranean Journal of Communication, 8(1), 121-135. doi: https://doi.org/10.14198/medcom2017.8.1.9

Van Engeland, A., \& Rudolph, R. (2016). From terrorism to politics. Abingdon-on-Thames: Routledge.

Watzlawick, P. (1979). ¿Es real la realidad? Confusión, Desinformación, Comunicación. Barcelona: Heider.

Williams, A. \& Baláž, V (2015). Tourism risk and uncertainty: Theoretical reflections. Journal of Travel Research, 54(3), 271-287.doi: https://doi.org/10.1177/0047287514523334

$\mathrm{Wu}, \mathrm{H} .(1998)$. Investigating the determinants of international news flow a meta-analysis. International Communication Gazette, 60 (6), 493-512. https://doi.org/10.1177/0016549298060006003 